\title{
Space structure of hadrons in elastic scattering at high energy
}

\section{P. Desgrolard 母}

Institut de Physique Nucléaire de Lyon, IN2P3-CNRS et Université Claude Bernard, 43 boulevard du 11 novembre 1918, F-69622 Villeurbanne Cedex, France

\begin{abstract}
s
We have confirmed and complemented previous conclusions from studies of the impact parameter profiles, relative to $p p$ and $\bar{p} p$ scattering, using a generalized eikonalized amplitude. In particular, the transition from the grey to the black disk is followed, when the energy increases beyond $\sim 3 \mathrm{TeV}$ and for the inelastic process, by a black ring surrounding the antishadow scattering mode at the center. The ranges of hadronic forces responsible for the elastic and inelastic processes are estimated by means of root-mean-squares of the impact parameter. Predictions at the LHC energy are explicitly given. The effect of eikonalization is discussed.
\end{abstract}

\section{Introduction}

Since the pionnering work of Amaldi and Schubert, more than twenty years ago, the impact parameter (or $b$ )-representation has a long history in the analysis of high-energy $p p$ and $\bar{p} p$ elastic scattering (e.g. see [洒] and references therein).

Beyond the discussion of the elastic profile and overlap function (e.g. [2]), of the shadow and antishadow scattering mode [3], extrapolations have also been investigated [4] at the forthcoming facilities of the CERN Large Hadron Collider (LHC).

Aside from these explorations, the following mean-squares of impact parameter have not enjoyed much popularity since their introduction [5]

$$
\begin{aligned}
<b^{2}(s)>_{t o t} & =\frac{\int_{0}^{\infty} b d b \cdot b^{2} \Im \mathrm{m} H(s, b)}{\int_{0}^{\infty} b d b \cdot \Im \mathrm{m} H(s, b)} \\
<b^{2}(s)>_{e l} & =\frac{\int_{0}^{\infty} b d b \cdot b^{2}|H(s, b)|^{2}}{\int_{0}^{\infty} b d b \cdot|H(s, b)|^{2}} \\
<b^{2}(s)>_{\text {in }} & =\frac{\int_{0}^{\infty} b d b \cdot b^{2} G_{i n}(s, b)}{\int_{0}^{\infty} b d b \cdot G_{i n}(s, b)}
\end{aligned}
$$

$H(s, b)$ is the elastic scattering amplitude in the $b$-representation (or profile function) at a center of mass energy $\sqrt{s}$ and $G_{i n}(s, b)$ the inelastic overlap function, sum of all inelastic channels contributions. The reason for this lack of interest up to recently [6] is probably due to the fact that it is often convenient and sufficient to discuss the three quantities $\Im \mathrm{m} H(s, b),|H(s, b)|^{2}, G_{i n}(s, b)$ versus $s$ and $b$.

\footnotetext{
${ }^{1}$ E-mail: desgrolard@ipnl.in2p3.fr
} 
From a fundamental point of view, it is certainly worth to discuss also the corresponding "root-mean-squares" of the impact parameters ( $b$-rms)

$$
\sqrt{<b^{2}(s)>_{t o t}}, \sqrt{<b^{2}(s)>_{e l}}, \sqrt{\left\langle b^{2}(s)>_{\text {in }}\right.},
$$

in terms of characteristics of the averaged ranges of hadronic forces responsible for elastic and inelastic collision processes. The estimation of the $b$-rms could provide a new light on a simple physical picture at very high energy, in particular at the LHC, where the antishadow scattering mode is expected to be a potential discovery (see [4] and references therein).

It is the aim of this note to complement a traditional study of the impact parameter elastic and inelastic profiles with a description of the space structure of hadrons in elastic scattering at high energy, especially of protons at LHC.

\section{Choice of an amplitude}

We note the definitions (1-3) are written in accordance with the notations used in the unitarity equation

$$
\Im \mathrm{m} H(s, b)=|H(s, b)|^{2}+G_{i n}(s, b),
$$

which integrated over $b$, running from zero to the infinity, gives simply the summation of the cross-sections: $\sigma_{\text {tot }}(s)=\sigma_{e l}(s)+\sigma_{i n}(s)$.

In order to estimate $H(s, b), G_{i n}(s, b)$ and the $b$-rms, we have chosen the dipole version of the generalized eikonalized (GE) scattering amplitude found in [7] for both $p p$ and $\bar{p} p$ elastic scattering processes. It concerns a generalization of the ordinary eikonal method, constrained by unitarity [8], taking into account all intermediate multiparticle states involving the crossing-even and crossing-odd combinations of Reggeons. The formalism involves three parameters, $\lambda_{0}, \lambda_{+}, \lambda_{-}$, corresponding to the three possible intermediate states. At the Born level the amplitude involves two secondary Reggeons, constructed from a simple Regge pole in the angular momentum $j$-plane, a Pomeron-" dipole", linear combination of a simple with a double pole and finally an Oddderon-dipole including a factor for damping at $t=0$.

The reasons for such a choice are:

- it is a recently published amplitude, implying the most general (to our knowledge) treatment of the eikonalization procedure;

- it leads to a very satisfying fit up to the largest explored 4-momentum transfer squared $\left(q^{2}=-t=14 \mathrm{GeV}^{2}\right.$, neigbouring with the limit of validity of the Regge theory at $\sqrt{s}=27 \mathrm{GeV})$ and up to the Tevatron energy $(1800 \mathrm{GeV}) \mathrm{H}$.

${ }^{2}$ The $p p$ cosmic rays data up to $40 \mathrm{TeV}$ were of no help in the fit because of their too large errors, but they are also reproduced by the model. The prediction of the total cross-sectiom at the LHC $\sigma_{\text {tot }}=107 \mathrm{mb}$ agrees with most common predictions in the literature (e.g., see [9]). 
We add that the effect of eikonalizing the Born amplitude, on the profiles and on the $b$-rms and related quantities, may be easily evaluated. We notice that the present nuclear amplitude [7], that we use, involves an eikonal unitarization while in [4, 10], that we discuss, a $U$-matrix unitarization has been assumed.

In our opinion, the choice of an amplitude, respecting the Froissart-Martin bound and accounting data available in the widest range of high energies and transfers is crucial to get confidence on predictive power outside the fitted sets of data. The CERN LHC at $\sqrt{s}=14 \mathrm{TeV}$ - and also the BNL Relativistic Heavy Ion Collider (RHIC) at a lower energy $\sim 500 \mathrm{GeV}$ - is probably the most interesting case to discuss presently (remember e.g. the accepted project [11] plans LHC measurements up to $\left.|t|=10 \mathrm{GeV}^{2}\right)$.

\section{$3 \quad$ Results and discussion}

Firstly, in order to test and generalize the model independence of the main conclusions already stated in [10, we performed a similar study, but with the Dipole GE amplitude of [7]. We reproduced similar results concerning $\Im \mathrm{m} H(s, b)$ and $G_{i n}(s, b)$, versus $b$, up to the highest energies.

1. An example is given Table 1 for the central opacity of the nucleon $\Im m H(s, 0)$. The agreement is good with existing experimental values. Prediction is also given for the LHC (where the black disk limit is overtaken)

\begin{tabular}{|c||c|c|c|c|}
\hline Energy $(\mathrm{GeV})$ & $\begin{array}{c}53 \\
(\mathrm{ISR})\end{array}$ & $\begin{array}{c}546 \\
(\mathrm{~S} \bar{p} p \mathrm{~S})\end{array}$ & $\begin{array}{c}1800 \\
(\text { Tevatron })\end{array}$ & $\begin{array}{c}14000 \\
(\mathrm{LHC})\end{array}$ \\
\hline Exp. & 0.360 & $0.420 \pm 0.004$ & $0.492 \pm 0.008$ & - \\
Calc. & 0.368 & 0.435 & 0.488 & 0.592 \\
\hline
\end{tabular}

Table 1: Central opacity of the nucleon, $\Im \mathrm{m} H(s, 0)$, calculated with the Dipole GE amplitude of [7] and compared to available experimental values [12] .

2. In qualitative agreement with «4, 10], we confirm that $\Im \mathrm{m} H(s, b)$, respecting the unitarity limit 1, remains central all the way, exceeding the black disk limit $1 / 2$ above the threshold calculated value

$$
\sqrt{s} \sim 3 \mathrm{TeV}
$$

and then $G_{i n}(s, b)$ undergoes a transition : from central it becomes peripheral. In other terms we find that, beyond this threshold, its maximum $(=1 / 4)$ is shifted from $b=0$ towards increasing $b$. The "interaction radius" $(R=R(s))$, where the maximal absorption takes place is e.g. $R \sim .6 \mathrm{fm}$ at $14 \mathrm{TeV}$ (see Fig.1) and would smoothly becomes $R \sim 1.2 \mathrm{fm}$ at $1000 \mathrm{TeV}$. We also find a hollow appearing at the center in $G_{i n}(s, b)$ becoming deeper and deeper when $s$ increases: e.g. 0.23 at $14 \mathrm{TeV}$ (Fig.1), 0.15 at $1000 \mathrm{TeV}$. 
3. It is a consequence of the chosen model [7, \&] that $H(s \rightarrow \infty, b) \rightarrow \frac{i}{2 \lambda_{+}}$, with $\lambda_{+}=0.5$, respecting the unitarity inequality $|H(s, b)| \leq 1$, dictates at the center $\Im \mathrm{m} H(s \rightarrow \infty, b=0) \rightarrow 1, G_{i n}(s \rightarrow \infty, b=0) \rightarrow 0$.

4. Thus, from our calculations, the appearance of the antishadow mode reveals at small values of $b$, in a natural way at a high energy, in conformity with its original introduction [3] and further studies (e.g. [4, 10). It is worth emphazing that the antishadow scattering mode is predicted here using a hadron-hadron amplitude quite different from those used in other calculations. This enforces the model independence of such a prediction but of course only experiments at very high energy could decide whether it exists or no. We found a calculated energy threshold for its apparition above the Tevatron, but below the LHC 3 .

5. It is interesting to mention the effect of eikonalization by changing $H, G_{i n}(s, b)$, once eikonalized and fitted, into $h, g_{i n}(s, b)$, at the Born level and non-refitted. Returning to the Born level brings forwards the antishadow mode characterics i.e. minoration of the energy threshold and majoration of the interaction radius. The drastic effect of eikonalization, well known on the currently fitted observables, is clearly visible, at the LHC energy for example, on $\Im m H$ and $G_{\text {in }}$ (see Fig.1): the antishadow scattering holds at $b \lesssim 0.6 \mathrm{fm}$ after eikonalization and at $b \lesssim 0.9 \mathrm{fm}$ at the Born level (these values correspond to the maximum $1 / 4$ taken by $G_{i n}$ and to the crossing of the black disk limit $1 / 2$ by sm $H$ at the LHC).

Secondly, in order to complete the preceding study, we have also calculated the three (total, elastic and inelastic) root-mean-squares of the impact parameter, defined in (4) and interpreted as characteristics of the ranges of hadron forces responsible for corresponding collisions. There are plotted in Fig.2, versus $\sqrt{s}$, at the Born level and after the generalized eikonalization.

The main remarks suggested by these curves are in order

1. A common gross characteristic of the $b$-rms is to have a mild dependence on the process and on the energy beyond the ISR up to the LHC. Roughly speaking, they lie between 0.7 fermi and 1.2 fermi.

2. They show almost parallel and linear increases with the energy.

3. To be more precise $\sqrt{\left.<b^{2}(s)\right\rangle_{\text {tot }}}$ goes from 0.9 to $1.2 \mathrm{fm} ; \sqrt{\left\langle b^{2}(s)\right\rangle_{\text {el }}}$ goes from 0.6 to $0.9 \mathrm{fm} ; \sqrt{\left\langle b^{2}(s)\right\rangle_{\text {in }}}$ goes from 1.0 to $1.3 \mathrm{fm}$, when the energy increases through four decades from $10 \mathrm{GeV}$ to $100 \mathrm{TeV}$.

4. Strictly speaking, the effect of eikonalization is poor on these quantities, in contrast with such effect on current observables (e.g. cross-sections) or on the elastic profile or on the inelastic overlap function, but in agreement with their

\footnotetext{
${ }^{3}$ It has been estimated $2 \mathrm{TeV}$ in [4] and $6 \mathrm{TeV}$ in [10], to be compared to $3 \mathrm{TeV}$ here.
} 
definitions as averages in the $b$ representation which smoothen the differences. The two first pairs of curves (total and elastic) yield almost parallel increases with the energy, the GE case being above the Born case, while the third one (inelastic) exhibits a Born $b$, close to GE $b$ and becoming greater at high energy. In other terms the inelastic Born line crosses the GE line in the $\mathrm{TeV}$ region, this crossing corresponds to the threshold value of the antishadow mode and recalls the importance of neglecting the eikonalization on the inelastic function as discussed above.

The results shown in Fig.2 are in agreement with the results from an analysis 13 at $53 \mathrm{GeV}$ and $546 \mathrm{GeV}$, calculated in a central hypothesis, i.e. with a non-existent degree of peripherality (see Table 2), in spite of strong differences in constructing the nuclear amplitude. Furthermore, we note, that the agreement still holds for $\sqrt{\left.<b^{2}(s)\right\rangle_{\text {tot }}}$ when a degree of peripherality is entered in the amplitude. This conforts us in the model independence of the present results.

\begin{tabular}{|c||c|c|c|c||c|c|}
\hline \multicolumn{1}{|c||}{ Energy $(\mathrm{GeV})$} & \multicolumn{2}{c|}{53} & \multicolumn{2}{c||}{541} & 1800 & 14000 \\
\hline & P.W. & {$[13]$} & P.W & {$[13]$} & P.W & P.W \\
\hline$\sqrt{\left.<b^{2}\right\rangle_{\text {tot }}}(\mathrm{fm})$ & 1.00 & 1.03 & 1.04 & 1.13 & 1.07 & 1.14 \\
$\sqrt{\left.<b^{2}\right\rangle_{\text {el }}}(\mathrm{fm})$ & 0.67 & 0.68 & 0.72 & 0.76 & 0.76 & 0.83 \\
$\sqrt{\left.<b^{2}\right\rangle_{\text {in }}}(\mathrm{fm})$ & 1.06 & 1.09 & 1.11 & 1.21 & 1.16 & 1.26 \\
\hline
\end{tabular}

Table 2: Total, elastic, inelastic root-mean-squares calculated with the GE amplitude of [7], at four representative energies, in this present work (P.W). The values of [13] for the two lowest energies, evaluated in a central hypothesis, are also quoted.

\section{Conclusion}

In this note, we have used an amplitude [7] fitted, after a generalized eikonalization process, reproducing all $p p$ and $\bar{p} p$ elastic scattering data. We have confirmed and completed conclusions of previous papers [4, 10] from a study of the impact parameter profiles for elastic and inelastic processes. They concern in particular the transition from the grey to the black disk, which is expected to be followed by the inelastic overlap function and when the energy increases beyond a threshold at $\sim 3 \mathrm{TeV}$, by a black ring surrounding the antishadow scattering mode at the center $(b=0)$.

According to this scenario, the old picture of a proton getting "BEL" (blacker, edgier, larger) in $p p$ and $\bar{p} p$ collisions, when the energy increases, still holds for both elastic and inelastic processes below the threshold. Above (let us say in the region of LHC energies), the antishadow mode appears in the inelastic collisions for which the proton behaves like a half-transparent core (a grey disk), with an outer shell (a black ring), mainly responsible for inelastic process. No echo is found on the traditional behaviour of the proton in the elastic and total processes. 
The ranges of hadronic forces responsible for elastic and inelastic processes, estimated by means of root-mean-squares of the impact parameter are found respectively $\sim 0.8 \mathrm{fm}$ and $\sim 1.3 \mathrm{fm}$, at LHC energy. They are only weakly dependent of the eikonalization, enforcing the interest of the Born approximation when estimating such characteristics.

Acknowledgements. I would like to thank V. Kundrat, S. Troshin, N. Tyurin for useful comments and E. Martynov for a critical reading of the manuscript.

\section{References}

[1] U. Amaldi, K. R. Schubert, Nucl. Phys. B 166, 301 (1980)

[2] R. Henzi, P. Valin, Phys. Lett. B 132, 443 (1983)

[3] S.M. Troshin, N.E.Tyurin, Phys. Lett. B 316, 175 (1993)

[4] S.M. Troshin, N.E. Tyurin, arXiv:hep-ph/0004084

S.M. Troshin, N.E. Tyurin, in Proceedings of the International Conference (VIIIth Blois Workshop) on Elastic and Diffractive Scattering, edited by V.A. Petrov and A.V.Prokudin, (World Scientific, Singapore, 2000), p.51;

V.A. Petrov, A.V. Prokudin, S.M. Troshin, N.E. Tyurin, arXiv:hep-ph/0103257

[5] V. F. Edneral, S. M. Troshin, N. E. Tyurin, Sov. J. Nucl. Phys. 30, 577 (1979)

[6] V. Kundrát, M. Lokajíceek, D. Krupa, in Proceedings of the International Conference ("HS98") on Hadron Structure, edited by D. Bruncko, P. Strizenec, (Kosice, Slovak. Acad. Sci., Inst. Exp. Phys., 1998) p.389

[7] P. Desgrolard, M. Giffon, E. Martynov, E. Predazzi, Eur. Phys. J. C 16, 499 $(2000)$

[8] P. Desgrolard, M. Giffon, E. Martynov, E. Predazzi, Eur. Phys. J. C 14, 683 (2000)

[9] M.M. Block, F. Halzen, T. Stanev, Phys. Rev. Lett. 83, 4926 (1999);

see also R.F. Avila, E.G.S. Luna, M.J. Menon, arXiv:hep-ph/0105065, where distinct scenarios are investigated for $p p$ at the highest energies

[10] P. Desgrolard, L, Jenkovszky, B. Struminsky, Eur. Phys. J. C 11, 145 (1999)

[11] TOTEM collaboration, Technical proposal, CERN/LHCC 97-49 (1997) and 99$7(1999)$

[12] R. Castaldi, G. Sanguinetti, Ann. Rev. Nucl. Part. Sci. 35, 351 (1985);

S. Abe et al. , CDF Collaboration, Phys. Rev. D 50, 550 (1994); 
P. Giromini, CDF Collaboration, in Proceedings of International Conference on Elastic and Diffractive Scattering (V th Blois workshop), edited by H.M. Fried, K. Kang, C-I. Tan (Word Scientific, Singapore, 1993), p.30

[13] V. Kundrát, M. Lokajíc̃ek, D. Krupa, arXiv:hep-ph/0001047

V. Kundrát, M. Lokajíc̃ek, D. Krupa, in Proceedings of the International Conference (VIII th Blois Workshop) on Elastic and Diffractive Scattering, edited by V.A. Petrov and A.V.Prokudin, (World Scientific, Singapore, 2000), p.333 


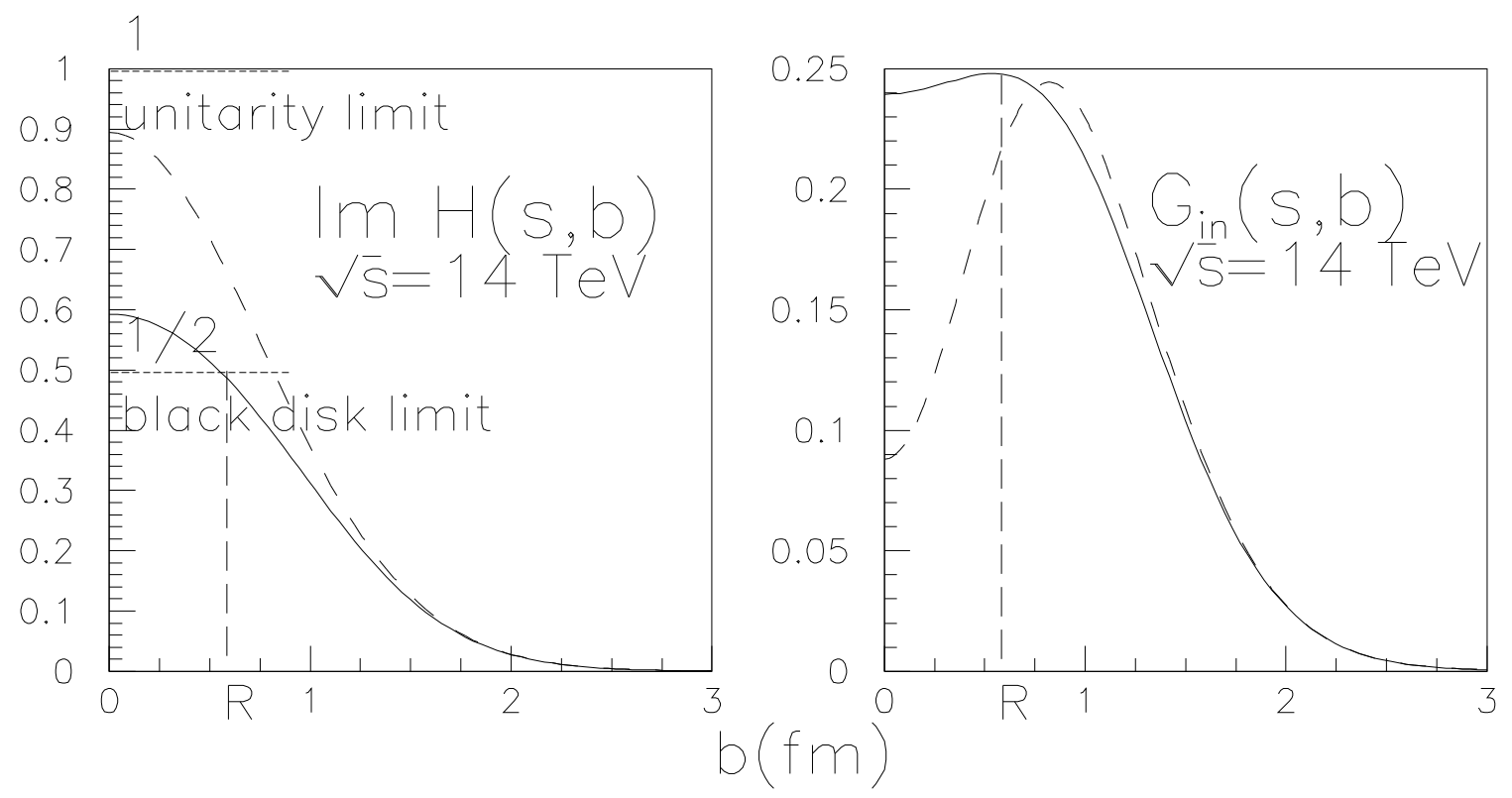

Figure 1. $\Im \mathrm{m} H(s, b)$ and inelastic overlap function $G_{i n}(s, b)$ versus the impact parameter $b$ calculated at the LHC energy in the GE formalism, with the fitted amplitude of [7] for the $\bar{p} p$ elastic scattering (solid line). $R$ is the "interaction radius" (see the text). A comparaison is shown with the -non-fitted- Born predictions (dashed line), proving the effect of eikonalization which soffens the antishadow characteristics.
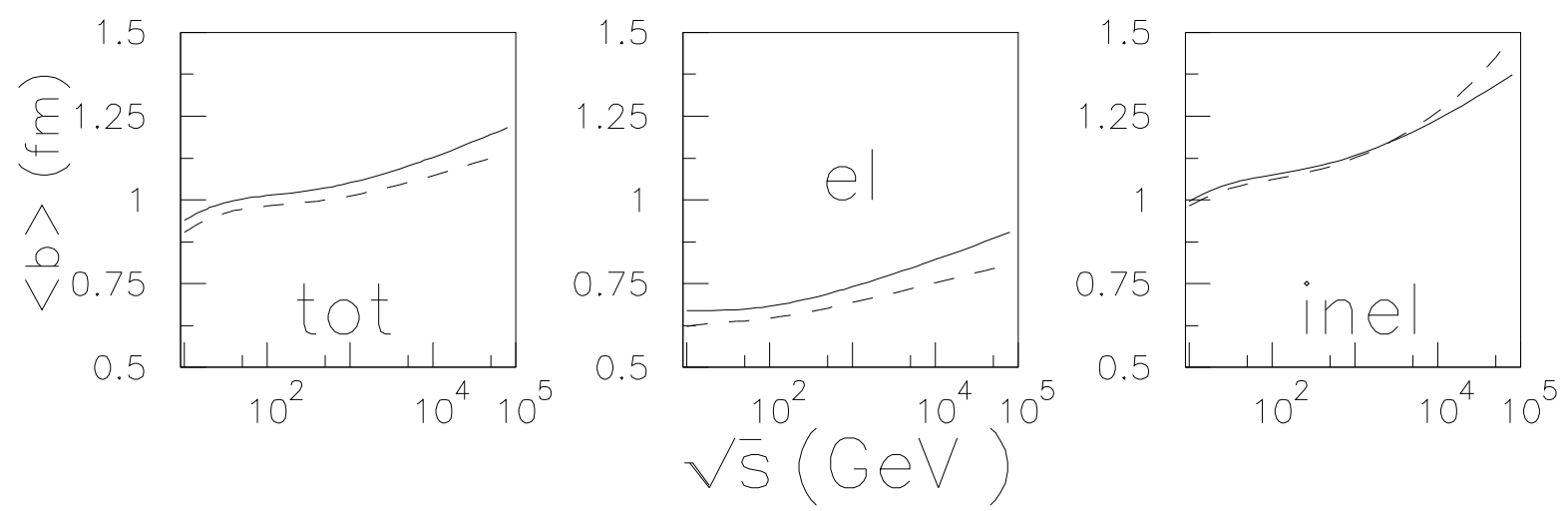

Figure 2. Total, elastic, inelastic root-mean-squares of impact parameter (5) calculated in the GE formalism, with the fitted amplitude of [7] for the $\bar{p} p$ elastic scattering (solid line, the $p p$ plots would be indiscernable). A comparaison is shown with the Born predictions (dashed line), proving the small effect of eikonalization on these quantities. 